\title{
BMJ Open Supplementation with Bifidobacterium longum subspecies infantis EVC001 for mitigation of type 1 diabetes autoimmunity: the GPPAD-SINT1A randomised controlled trial protocol
}

Anette-Gabriele Ziegler, ${ }^{1,2}$ Stefanie Arnolds, ${ }^{1}$ Annika Kölln, ${ }^{1}$ Peter Achenbach, ${ }^{1,2}$ Reinhard Berner, ${ }^{3}$ Ezio Bonifacio, ${ }^{4}$ Kristina Casteels, ${ }^{5,6}$ Helena Elding Larsson, ${ }^{7,8}$ Melanie Gündert (D) , Joerg Hasford, ${ }^{9}$ Olga Kordonouri, ${ }^{10}$ Markus Lundgren, ${ }^{7}$ Mariusz Oltarzewski, ${ }^{11}$ Marcin L Pekalski, ${ }^{12}$ Markus Pfirrmann, ${ }^{9}$ Matthew D Snape, ${ }^{13,14}$ Agnieszka Szypowska, ${ }^{15}$ John A Todd, ${ }^{12}$ GPPAD STUDY GROUP

To cite: Ziegler A-G, Arnolds S, Kölln A, et al. Supplementation with Bifidobacterium longum subspecies infantis EVCO01 for mitigation of type 1 diabetes autoimmunity: the GPPADSINT1A randomised controlled trial protocol. BMJ Open 2021;11:e052449. doi:10.1136/ bmjopen-2021-052449

- Prepublication history and additional supplemental material for this paper are available online. To view these files, please visit the journal online (http://dx.doi.org/10.1136/ bmjopen-2021-052449).

Received 19 April 2021 Accepted 08 October 2021

D) Check for updates

(c) Author(s) (or their employer(s)) 2021. Re-use permitted under CC BY-NC. No commercial re-use. See rights and permissions. Published by BMJ.

For numbered affiliations see end of article.

\section{Correspondence to} Dr Anette-Gabriele Ziegler; anette-g.ziegler@helmholtzmuenchen.de

\section{ABSTRACT}

Introduction The Global Platform for the Prevention of Autoimmune Diabetes-SINT1A Study is designed as a randomised, placebo-controlled, double-blind, multicentre, multinational, primary prevention study aiming to assess whether daily administration of Bifidobacterium infantis from age 7 days to 6 weeks until age 12 months to children with elevated genetic risk for type 1 diabetes reduces the cumulative incidence of beta-cell autoantibodies in childhood.

Methods and analysis Infants aged 7 days to 6 weeks from Germany, Poland, Belgium, UK and Sweden are eligible for study participation if they have a $>10.0 \%$ expected risk for developing multiple beta-cell autoantibodies by age 6 years as determined by genetic risk score or family history and HLA genotype. Infants are randomised 1:1 to daily administration of $B$. infantis EVC001 or placebo until age 12 months and followed for a maximum of 5.5 years thereafter. The primary outcome is the development of persistent confirmed multiple beta-cell autoantibodies. Secondary outcomes are (1) Any persistent confirmed beta-cell autoantibody, defined as at least one confirmed autoantibody in two consecutive samples, including insulin autoantibodies, glutamic acid decarboxylase, islet tyrosine phosphatase 2 or zinc transporter 8, (2) Diabetes, (3) Transglutaminase autoantibodies associated with coeliac disease, (4) Respiratory infection rate in first year of life during supplementation and (5) Safety. Exploratory outcomes include allergy, antibody response to vaccines, alterations of the gut microbiome or blood metabolome, stool pH and calprotectin.

Ethics and dissemination The study was approved by the local ethical committees of the Technical University Munich, Medical Faculty, the Technische Universität Dresden, the Medizinische Hochschule Hannover, the Medical University of Warsaw, EC Research UZ Leuven and the Swedish ethical review authority. The results will be disseminated through peer-reviewed journals and

\section{Strengths and limitations of this study}

This is the first adequately powered placebo controlled study to test the supplementation with Bifidobacterium infantis for mitigation of type 1 diabetes autoimmunity.

- Includes other health outcomes such as coeliac autoimmunity, respiratory infections, allergy, antibody response (IgG titres) to vaccines, alterations of the gut microbiome or blood metabolome, measurement of stool pH and calprotectin.

- The requirement to identify eligible at-risk infants by genetic screening does not allow to introduce $B$. infantis supplementation in the first days of life or during pregnancy, when it may be most beneficial.

- There could be advantages in using multiple probiotic strains with complementary metabolic capacities.

- It may have been advantageous to add a prebiotic to the $B$. infantis formulation to compensate for the possibility that some mothers stopped breast feeding early in the trial.

conference presentations and will be openly shared after completion of the study.

Trial registration number NCT04769037.

\section{INTRODUCTION}

Type 1 diabetes (T1D) results from an immune-mediated destruction of the pancreatic islet beta-cells resulting in insulin deficiency. This process is clinically silent and can be identified by circulating autoantibodies to beta-cell antigens (glutamic acid decarboxylase (GADA), islet tyrosine phosphatase 2 (IA-2A), insulin autoantibodies (IAA) and zinc transporter 8 (ZnT8A). ${ }^{1}$ Beta-cell 
autoantibodies occur early in life with a peak incidence period between age 9 months and 3 years, ${ }^{2-4}$ and the risk to develop multiple beta-cell autoantibodies exponentially declines with age ${ }^{56}$ On the basis of these findings, it is concluded that any interventional therapy given as a primary prevention strategy must be started early in life.

This randomised, placebo-controlled study will evaluate whether supplementation with a daily dose of a probiotic in the first year of life can reduce the risk of developing beta-cell autoimmunity in children identified by the Global Platform for the Prevention of Autoimmune Diabetes (GPPAD) as being genetically at risk for developing T1D (previously described in detail in 7 and 8 ). The rationale for this study combines the most recent findings concerning the specific role that the commensal bacteria, microbiota and their genes, the microbiome, could play in the induction of peripheral tolerance to insulin, ${ }^{9-12}$ and builds on knowledge of the reported health and clinical benefits of early probiotic supplementation in peanut allergy prevention ${ }^{13}$ and in lowering mortality owing to sepsis in children. ${ }^{14}$ Although it is widely accepted that the induction of the state of tolerance to beneficial bacteria during early life is critical for a newborn's survival, the clinical benefit for the prevention of immune-related diseases is only now being explored. It is believed that tolerance is achieved when the innate and adaptive immune cells promote tolerogenic immune responses to dietary and commensal antigens as well as reactions to a variety of beneficial metabolites produced by commensal microbes, in particular the short chain fatty acids (SCFAs) ${ }^{15-19}$ The SINT1A study follows the ongoing Primary Oral Insulin Trial (POInT), ${ }^{8}$ which administers insulin orally to train and sensitise the immune system at an early stage via gut exposure so that autoimmunity against insulin does not occur.

\section{Evidence for dysbiosis in children prior to the development of T1D}

Evidence that microbiome changes may alter the risk for T1D is presented by a number of prospective studies which have shown that changes in the microbiome precede the development of beta-cell autoimmunity and T1D. These include the BABYDIET study where alterations in microbial interaction networks were observed at age 0.5 and 2 years in children who developed beta-cell autoimmunity, ${ }^{9}$ and studies from Finland where higher abundances of Bacteroides dore $^{20}$ and a decrease in microbial diversity were described in children with genetic predisposition to T1D. ${ }^{21}$ The TEDDY study confirmed these alterations and found that the microbiome of children who did not develop betacell autoimmunity contained more genes that were related to fermentation and the biosynthesis of SCFAs supporting the protective effects of SCFA in early-onset human T1D. ${ }^{10}$ Furthermore, the TEDDY study has reported that probiotic supplementation in the first 27 days of life, and only in this early period (documented by questionnaires and diary records) was associated with a decreased risk of beta-cell autoimmunity providing the first evidence that correcting dysbiosis in infants may be beneficial for children at risk of T1D. ${ }^{11}$ A recent finding links microbial metabolism to insulin-specific immune responses: the microbial enzymes belonging to the transketolase superfamily contain the primary insulin autoantigenic epitope (INS B:9-25). The microbial transketolase upregulation reflects the adaptation of the microbiome to digest sugar polymers during weaning and matches the time of autoantibody appearance against insulin. It has been suggested that an immune response to insulin mimotopes due to commensal dysbiosis is a possible primary cause of T1D. ${ }^{12}$

Altogether, these results suggest that correcting dysbiosis in early life could help to promote immune tolerance and thus inhibit the initiation of beta-cell autoimmunity.

\section{Previous clinical studies using Bifidobacterium infantis in children}

A substantial body of evidence has connected gut inflammation with improper immune programming and the subsequent development of autoimmune conditions including T1D, atopic dermatitis, food allergies and asthma. ${ }^{1922}$ Bifidobacteria and in particular Bifidobacterium longum subspecies infantis (Bifidobacterium infantis) have positive properties that potentially counteract the development of gut inflammation in the first few months of life. ${ }^{23}{ }^{24}$ Depleted numbers of Bifidobacteria are associated with immune-related diseases such as allergy. ${ }^{256}$ Bacteroides species, which are naturally outcompeted by Bifidobacteria, are present at higher numbers within the microbiota of children with high susceptibility to autoimmunity. ${ }^{19}$ There is additional evidence that Bifidobacterium abundance in early infancy increases protective efficacy of vaccines by enhancing immunological memory. ${ }^{27}$ Lactic acid bacteria and bifidobacteria are increasingly administered to pregnant women and infants with the intention of improving health. A number of clinical studies have been conducted to document the safety and health benefits of dietary supplementation with bacterial strains (probiotics). Previous clinical studies using B. infantis are summarised in table 1 .

These studies show first evidence that supplementation with $B$. infantis is safe. ${ }^{28-30}$ The IMPRINT (Infant Microbiota and Probiotic Intake) study demonstrates that supplementation with $B$. infantis $\left(1.8-2.8 \times 10^{10}\right.$ colony forming units (CFU)) for 14 days (from day 7 to day 21) is well tolerated. ${ }^{28}$ Stools are fewer and better formed in infants in the supplementation group compared with the non-supplemented group. A follow-up study indicates that supplementation results in significant changes of the faecal microbiome composition ${ }^{29}$ including evidence of persistent colonisation of the probiotic organism. Infants colonised by Bifidobacteriaceae have fourfold-lower faecal endotoxin levels, consistent with observed lower levels of Gram-negative Proteobacteria and Bacteroidetes, lower stool $\mathrm{pH}$ and higher faecal concentrations of SCFAs. ${ }^{29}$ In addition, proinflammatory cytokines are significantly lower in B. infantis-fed infants. ${ }^{30}$ The mentioned results suggest that correcting dysbiosis in early life could help 
Table 1 Clinical studies on Bifidobacterium infantis

\begin{tabular}{lll}
\hline Reference & No of participants* & Main results \\
\hline 28 & 80 & $\begin{array}{l}\text { Safe consumption and good tolerance of B. infantis; stools significantly fewer and } \\
\text { better formed. }\end{array}$ \\
29 & 66 & $\begin{array}{l}\text { Significant changes to faecal microbiome composition; colonisation with } B \text {. infantis; } \\
\text { higher abundance of faecal short chain fatty acids; lower stool pH. } \\
\text { Lower faecal calprotectin levels; lower enteric inflammation. }\end{array}$ \\
\hline
\end{tabular}

${ }^{*}$ Participants in total, meaning the group of children $B$. infantis fed and the group placebo fed (for all: treatment from day 7 on, dose 1.8$2.8 \times 10^{10}$ colony forming units), all participants were breastfed infants.

to promote immune tolerance and thus inhibit the initiation of beta-cell autoimmunity. In this context, a supplementation with $B$. infantis seems promising.

\section{Primary objective}

To determine whether daily administration of B. infantis EVC001 from age 7 days to 6 weeks until age 12 months to children with elevated genetic risk for T1D reduces the cumulative incidence of beta-cell autoantibodies in childhood.

\section{METHODS}

\section{Outcome measures}

Primary outcome

The primary outcome of SINT1A is the elapsed time from random treatment assignment to the development of persistent confirmed multiple beta-cell autoantibodies. For subjects who developed persistent confirmed multiple beta-cell autoantibodies, the elapsed time will be from the random treatment assignment to the first confirmed autoantibody positive sample used in defining the persistent confirmed multiple beta-cell autoantibody positive status. It is expected that beta-cell autoantibodies will be detected prior to T1D diagnosis; however, the presence of diabetes in the absence of multiple beta-cell autoantibodies is also considered as a primary outcome endpoint, and in this case, the date of diagnosis is the time of the end point.

The study primary outcome is realised with either persistent confirmed multiple beta-cell autoantibodies or Oral Glucose Tolerance Test (OGTT) criteria for diabetes or clinical criteria for diabetes.

\section{Criteria for persistent confirmed beta-cell autoantibodies}

Criteria are based on the measurement of beta-cell autoantibodies against insulin (IAA), GAD65 (GADA), IA-2 (IA-2A) and ZnT8 (ZnT8A) tested in the GPPAD central autoantibody laboratory and, if positive, confirmed in the GPPAD confirmatory laboratory.

Confirmed IAA is defined as sample positive for IAA in both the GPPAD central and confirmatory laboratories. Confirmed GADA is defined as sample positive for GADA in both the GPPAD central and confirmatory laboratories. Confirmed IA-2A is defined as sample positive for IA-2A in both the GPPAD central and confirmatory laboratories. Confirmed ZnT8A is defined as sample positive for ZnT8RA or ZnT8WA in both the GPPAD central and confirmatory laboratories.

The status persistent confirmed beta-cell autoantibodypositive is defined as confirmed IAA, confirmed GADA, confirmed IA-2A or confirmed ZnT8A in two consecutive samples. Persistent confirmed multiple beta-cell autoantibodies (primary outcome) is defined as confirmed IAA, confirmed GADA, confirmed IA-2A or confirmed ZnT8A in two consecutive samples, AND at least one other confirmed antibody from these four antibodies in one sample. Persistent confirmed beta-cell autoantibodies that are considered maternally derived are NOT included as positive for the primary outcome.

\section{Criteria for T1D diagnosis}

Diabetes may be diagnosed in a small number of children before a persistent confirmed multiple islet autoantibody positive status is achieved as the multiple autoantibody outcome requires two consecutive positive samples. In these cases, the primary outcome status is assigned to the child.

Criteria for T1D diagnosis are, as defined by the American Diabetes Association, based on glucose testing, or the presence of unequivocal hyperglycaemia with acute metabolic decompensation (diabetic ketoacidosis). One of the following criteria must be met on two occasions as soon as possible but no less than 1 day apart for diabetes to be defined:

1. Symptoms of diabetes and a casual plasma glucose (PG) $\geq 200 \mathrm{mg} / \mathrm{dL}(11.1 \mathrm{mmol} / \mathrm{L})$.

Casual is defined as any time of day without regard to time since last meal. The classic symptoms of diabetes include polyuria, polydipsia and unexplained weight loss.

\section{OR}

2. Fasting PG (FPG) $\geq 126 \mathrm{mg} / \mathrm{dL}(7 \mathrm{mmol} / \mathrm{L})$. Fasting is defined as no caloric intake for at least 8 hours.

OR

3. Two-hour $\mathrm{PG} \geq 200 \mathrm{mg} / \mathrm{dL}(11.1 \mathrm{mmol} / \mathrm{L})$ during an OGTT. The test should be performed using a glucose load containing the equivalent of $1.75 \mathrm{~g} / \mathrm{kg}$ body weight to a maximum of $75 \mathrm{~g}$ anhydrous glucose dissolved in water. It is preferred that at least one of the two testing occasions involve an OGTT. 
Cases diagnosed with T1D will be adjudicated by the Endpoint Committee. Study participation will be terminated if T1D is reached.

\section{Secondary outcomes}

Secondary outcomes of the study are: (1) The development of any persistent confirmed beta-cell autoantibody, defined as at least one confirmed autoantibody in two consecutive samples, including IAA, GADA, IA-2A or ZnT8A, (2) Diabetes, (3) The development of persistent confirmed transglutaminase antibodies associated with coeliac disease, defined as confirmed autoantibody in two consecutive samples, (4) Respiratory infection rate in first year of life during supplementation and (5) Safety.

\section{Exploratory outcomes}

The following exploratory outcomes may be assessed or in part assessed on a portion of the participants. They may not necessarily be included in the primary outcome analysis and publication: (1) Allergy, (2) Antibody response (IgG titres) to vaccines, (3) Alterations of the stool microbiome or (4) Blood metabolome, (5) Stool pH and (6) Stool calprotectin concentration.

\section{Study design and organisation}

SINT1A will be conducted as an investigator-initiated, randomised, placebo-controlled, double-blind multicentre intervention study through GPPAD, a network of collaborating clinical study centres from European countries with sites in Belgium (Leuven), Germany (Dresden, Hannover, Munich), Poland (Warsaw), Sweden (Malmö) and UK (Newcastle, Cambridge). The Trial Coordinating Centre (GPPAD CC) is located at the Institute of Diabetes Research, Helmholtz Zentrum München. It manages coordination and communication between the SINT1A clinical study sites, and oversees the collection, analysis and storage of clinical data; also the supervision of regulatory activities, clinical research organisation activities, the manufacturer of the active supplement and the central laboratories is provided by the CC.

GPPAD was founded in 2015 with the aim to provide an international infrastructure to enable T1D primary prevention trials, identify infants with an elevated genetic risk of developing T1D and offer participation in randomised controlled trials aiming to reduce the incidence of T1D in children. ${ }^{8}{ }^{31}$ Until March 2021, more than 251000 infants have been screened and had their genetic risk of developing T1D evaluated using a combination of family history and 47 single-nucleotide polymorphisms (SNPs). ${ }^{7}$ From these, over 2000 infants have been identified as having a $10 \%$ probability or greater of developing multiple beta-cell autoantibodies by 6 years of age, making them eligible for GPPAD primary prevention trials. ${ }^{7}$ The first GPPAD trial POInT has now (March/2021) completed enrolment (1050 participants). ${ }^{8}$ SINT1A will commence in April 2021 with the first patient first visit.

\section{Study population}

Infants are tested for genetic risk of T1D based on advanced risk scores derived from 51 SNPs that define HLA-DR3, HLA-DR4, and HLA-DQ8 alleles as well as SNPs from HLA class I, and non-HLA T1D susceptibility genes, and from HLA class II protective alleles, as previously described. ${ }^{732}$ Infants with a predicted risk of $>10 \%$ to develop multiple beta-cell autoimmunity by age 6 years and who fulfil the inclusion criteria as stated below are eligible to participate in the GPPAD-SINT1A Study.

A total of 1144 infants will be enrolled and randomised 1:1 (B. infantis or placebo) in the SINT1A study (see figure 1). Children with T1D susceptible genotypes also have a marked risk of around 10\% for autoimmunity found in coeliac disease as shown in the TEDDY study. ${ }^{33}$

\section{Inclusion and exclusion criteria}

Participants must meet all entry criteria for the protocol as outlined below.

- Infants between the ages of 7 days and 6 weeks $(+14$ days in case of illness or COVID-19-related issues or unexpected delay in result reporting) at the time of randomisation.

- A $10 \%$ or higher genetic risk to develop multiple betacell autoantibodies by age 6 years

a. For infants without a first-degree family history of T1D, high genetic risk is defined as a DR3/ DR4-DQ8 or DR4-DQ8/DR4-DQ8 genotype and a Genetic Risk Score (GRS) that is in the upper 25th centile $(>14.4)^{34}$ or a DR3/DR4-DQ8 genotype with a GRS between the upper 50th (14.0) and 25th centile and a GG genotype at the rs3763305 SNP. These represent around $1 \%$ of all newborns.

b. For infants with a first-degree family history of type 1 diabetes, high genetic risk is defined as having HLA DR4 and DQ8, and none of the following protective alleles: DRB1*1501, DQB1*0503, DRB1*1303. These represent around $30 \%$ of infants with a firstdegree family history of T1D.

- Written informed consent signed by the custodial parent(s).

Participants may not enter the study if ANY of the following apply:

- Any medical condition, concomitant disease or treatment that may interfere with the assessments or may jeopardise the participant's safe participation in the study, as judged by the Investigators.

- Preterm delivery $<36$ weeks of gestation.

- Proven immunodeficiency.

- Any condition that could be associated with poor compliance.

- Diagnosis of diabetes at the time of recruitment.

\section{Informed consent}

The GPPAD-SINT1A Study will be described to the custodial parent(s) of potential participants by qualified GPPAD study personnel. The custodial parent(s) will have the opportunity to read the consent document and to discuss 


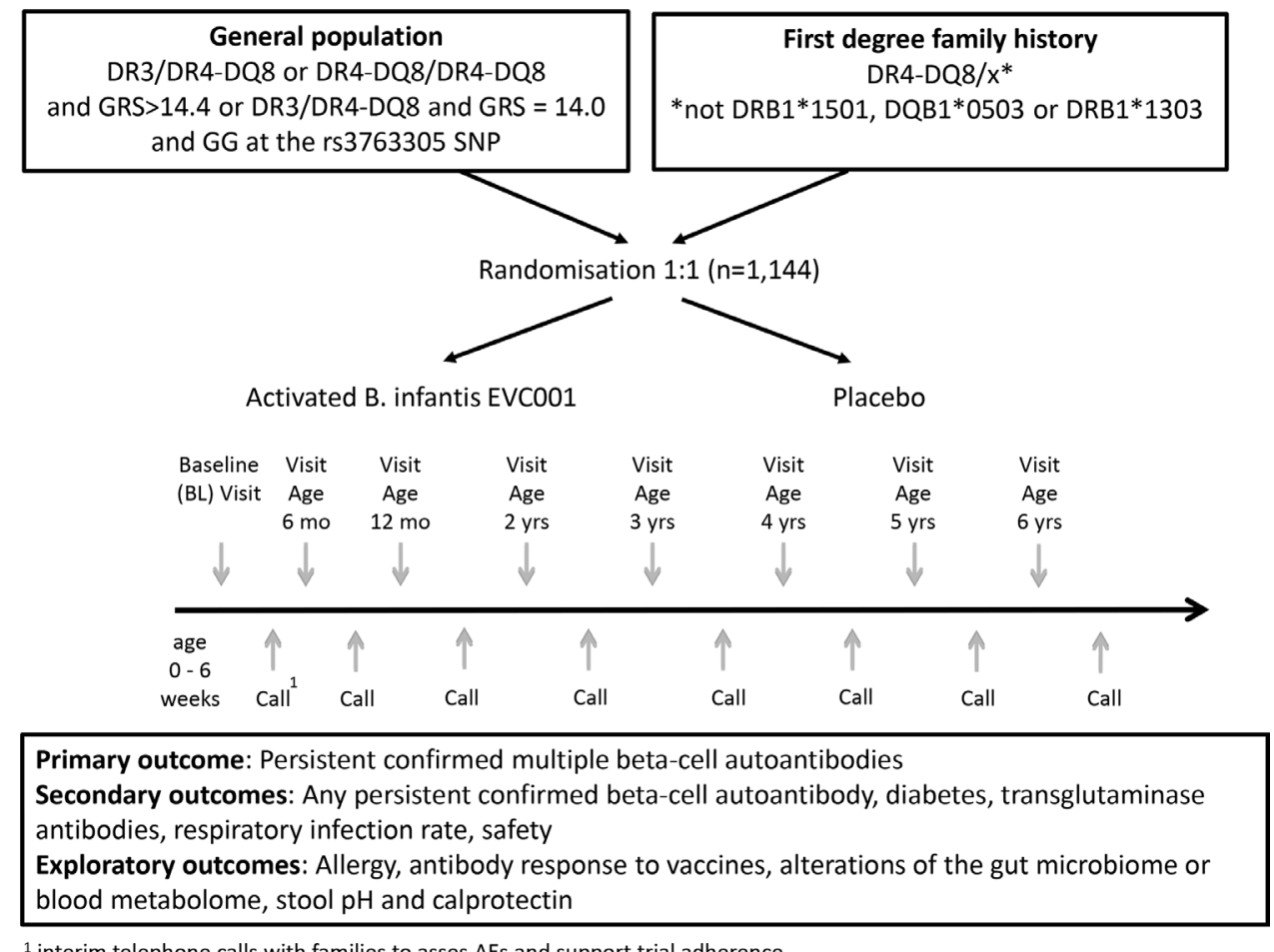

Figure 1 SINT1A study flow and time schedule for a participant with intervention until 12 months and maximum follow-up of 5.5 years. AEs, adverse events; GRS, Genetic Risk Score; SNPs, single-nucleotide polymorphisms.

any questions concerning the consent or study participation. The families will be given enough time to consider whether or not to participate. The custodial parent(s) will then be asked to sign and date an informed consent form prior to or at the baseline visit. Date and signature of the study Investigator (or other authorised study personnel, if applicable) will also be obtained on the consent form. A copy of the informed consent form will be handed out to the families. The custodial parent(s) of the prospective participant will be told that being in the study is voluntary and that the participant may withdraw from the study at any time, for any reason.

\section{Patient and public involvement}

Patients were not involved in the study design but in the prioritisation of the research question of T1D prevention. Patients support recruitment through dissemination, and participation in press conferences. Participating families will be informed about the outcome of the trial via webcast, letter and personal communication on the completion of the trial.

\section{Randomisation}

Subjects will be centrally randomised in a $1: 1$ ratio to one of the two intervention arms via Interactive Voice Response System/Interactive Web Response System (IVRS/IWRS) at the baseline visit. The participant and the treating physician and the central research team will be blinded. The study product packages will not indicate whether the content is B. infantis or placebo, but kit numbers. The IVRS/IWRS will assign the appropriate kit numbers for each participant following a randomisation list. Emergency unblinding will be available through the IVRS/helpdesk. Siblings within one household will be randomised to the same intervention arm to avoid mix-up of supplementation. Randomisation will be stratified for whether the child is still breastfed at the date of randomisation and study centre.

\section{Intervention}

Participants are randomised in a 1:1 ratio to receive either B. infantis or placebo. Each dose of the active supplement is provided as one sachet with $B$. infantis EVC001 at a minimum concentration of $8 \times 10^{9} \mathrm{CFU}$ in lactose. The dose was selected according to the previous IMPRINT study. ${ }^{28}$ The actual concentration as per batch certificate of analysis ranged from $13.8 \times 10^{9}$ to $15.8 \times 10^{9}$ CFU per sachet; the shelf-life is 15 months. The placebo consists of lactose, identical in appearance and taste to the active supplement. $B$. infantis/placebo will be administered orally, once a day, using single-dose sachets. It is recommended to administer the product in the morning (7:00-10:00 hours), preferably together with some breast milk. The content (powder) of the sachets is poured into a small bowl and mixed with $3-5 \mathrm{~mL}$ of breast milk, infant formula or water. The solution will be administered using a feeding syringe, preferably in the morning. Parent(s) will be instructed in the administration and storage of the sachets (should be kept frozen until use) at or prior to their baseline visit. The genome of $B$. infantis is available in the NCBI accession number NZ_LR655210 under the strain name USA001_1. ${ }^{35}$ Active and placebo products are provided by Evolve Biosystems, USA. Blinding, packing 
and distribution to clinical study sites is performed by the pharmacy, University of Heidelberg, Germany.

\section{Study timeline}

The study is expected to take 6.5 years to complete. This includes an intervention phase of 12 months, and minimum 2.5 (last participant enrolled) to maximum 5.5 years of follow-up (first participant enrolled) after intervention (figure 1). The enrolment period is projected to be 3.0 years.

\section{Study assessment}

The baseline visit includes the collection of information on medical history, C-section, breast feeding, proton pump inhibitor therapy, infections, probiotic use and antibiotic treatment prior to enrolment and the collection of a stool sample. Families will be instructed in the administration and storage of the blinded food product (B. infantis or placebo). Mothers will be encouraged to make their best effort to maintain breast feeding for at least the first 3-4 months, and they will be encouraged to continue breast feeding for as long as possible during the first year of the infant's life. Two more intervention visits (visit 2 and 3) are planned after 6 months and 12 months ( \pm 14 days). At these visits venous blood and a stool sample is collected, electronic questionnaires are discussed. The assessment of weight and height is performed at all visits. After the intervention period, study visit 4 will be conducted at age 2 years ( \pm 30 days), subsequent follow-up study visits will be conducted every year ( \pm 30 days) until the end of the study. A detailed table explaining study visits, and data and sample collection is shown in online supplemental file 1. All study relevant subject data and laboratory results are documented in corresponding electronic case report forms (eCRFs).

\section{E-diaries and allergy questionnaires}

Throughout the intervention period, parents will be asked to complete e-diaries fortnightly to collect information on breast feeding, respiratory infections, antibiotic treatment and treatment with proton pump inhibitors. Additional questionnaires to obtain information about allergies will be collected every 12 months starting at age 12 months \pm 14 days until the end of the study. The information given by the parents will be captured in a central database and reviewed and discussed during the study visits and phone calls between the visits.

\section{For participants who develop positive beta-cell or transglutaminase autoantibodies}

Participants who have confirmed positive beta-cell or transglutaminase autoantibodies during the study are asked to donate a confirmation sample within 4-12 weeks. If the participant has persistent confirmed beta-cell autoantibodies, the custodial parent(s) are informed and asked to participate in an educational programme informing about the diagnosis of beta-cell autoantibody positivity and possible symptoms of hyperglycaemic and metabolic decompensation. The child remains in the study and continues to be treated or followed as planned until the child has developed T1D or end of study. Home monitoring of blood glucose will be recommended 2-monthly if a child is considered at risk for a rapid progression to diabetes (eg, IA-2A positive, very high titres of antibodies or impaired blood glucose values). In case of persistent confirmed positivity for transglutaminase autoantibodies, an intestinal biopsy maybe recommended to clarify the diagnosis of coeliac disease. These children will continue to receive $B$. infantis/placebo and will be followed in the study for continued monitoring of diabetes development and safety assessments.

\section{Safety}

As the study intervention is not considered a medicinal product, safety reporting obligations as for IMP clinical trials do not apply. However, AEs and s (SAEs) up to 30 days after the last administration of the food product are assessed and captured in the eCRF. AEs will be graded as mild, moderate, severe, life-threatening or death according to the National Cancer Institute's Common Terminology Criteria for Adverse Events V.5.0.

Physical examinations, including measurement of height and weight, are performed at all visits.

\section{ANALYSIS}

All efficacy analyses will be conducted under the intentionto-treat principle whereby all effectiveness outcome data in all randomised subjects who have received at least one dose of $B$. infantis supplement or placebo will be included in all analyses as appropriate. Subjects who drop-out of the study will not be replaced. All data acquired prior to termination will be included in the primary analysis unless a participant withdraws consent.

\section{Primary outcome and analysis}

The cumulative incidence of multiple beta-cell autoantibodies over time since randomisation within each treatment group will be estimated from a Kaplan-Meier estimate of the 'beta-cell autoantibody-free' survival function. The difference between groups in the cumulative incidence functions, and the associated hazard functions, will be tested at the 0.05 level, two sided, using Cox regression including site as covariate. With respect to the primary outcome, the HR of $B$. infantis to placebo will be given together with its $95 \%$ CI. The final alpha is 0.05 (two sided). In case the assumptions of the sample size estimation hold, it will be possible to reject the null hypothesis of equal hazard rates with the power of $80 \%$, if 914 children will be uniformly randomised over 3 years and afterwards, all 914 children will be followed for another 3.5 years (6.5 years total duration after first enrollment). We have assumed a drop-out rate of $20 \%$, and therefore we need to randomise 1144 children to support an $80 \%$ power by a complete follow-up of 914 children ranging from 3.5 to 6.5 years. 


\section{Subgroup analyses of primary outcome}

It is reasonable to consider that $B$. infantis colonisation, breastfeeding status and secretor (FUT2 gene) status may impact the outcome. ${ }^{36}$ B. infantis colonisation will be measured in stool samples collected at visit age 3 months. A multiple Cox regression analysis will be performed on the primary outcome including treatment group and colonisation as a continuous variable. This will also be performed with a treatment group-colonisation interaction term and with colonisation as a categorical variable. Three categories representing no colonisation, low and high colonisation based on the distribution of values in the children will be used. Breast feeding will be encouraged. Nevertheless, a portion of the children will not be receiving breast milk when they start. Breast feeding will, therefore, be included as a categorical variable together with treatment in a Cox regression. An interaction analysis between colonisation and breastfeeding status will be performed, and a subgroup analysis for the primary outcome will be performed in children categorised by their FUT2 gene genotype as secretors and non-secretors. Additional variables that will be analysed by multivariable Cox regression include: (1) Children categorised as having a mother with diabetes versus the remainder; (2) Children who have HLA DR3/4-DQ8 versus the remainder; (3) Children who have the T1D susceptible INS AA genotype versus the remainder; (4) Sex; (5) Caesarean section versus vaginal birth; (6) body mass index at age 1 year as tertiles and (7) Genetic risk score tertiles.

\section{Secondary and exploratory outcomes and analyses}

For the secondary outcomes, the treatment arms will be compared on the corresponding incidence rates of each secondary outcome using the log rank statistic. Other secondary outcomes will be analysed by comparison between the $B$. infantis supplementation and placebo supplementation groups using appropriate statistics in dependence on the outcome of interest. Subgroup analyses analogous to those described for the beta-cell autoantibodies endpoint will be conducted on the secondary outcome endpoints. Exploratory analyses will examine the associations between $B$. infantis supplementation and allergy, mouth and stool organisms (microbiome), and blood markers such as the metabolome, lipidome, or inflammatory proteins and ancillary study measurements that specific sites may undertake.

\section{Study power and accrual target}

For the sample size estimation, the following scenario was chosen:

- Overall alpha level=0.05 (two sided).

- Overall beta level $=0.2$, that is, power $=0.8$.

- In the placebo group, at 3.5 years (approximate age of participants, 4 years), an event probability of $7.5 \%$ was assumed. Based on the exponential distribution, this leads to a hazard of 0.02227 .

- For the active treatment, it is assumed that the hazard rate will be halved.
- Accrual time is 3 years.

- Follow-up time is 3.5 years.

- A drop-out rate of $20 \%$ was taken into account.

The study has been designed to provide $80 \%$ power to detect a $50 \%$ risk reduction in the hazard rate of the event of confirmed persistent beta-cell autoantibodies using a two-sided test at the significance level 0.05 after 6.5 years of study duration. Decisive test will be the Wald test for the HR between the two groups within a Cox PH model. It is expected that the hazard is halved by active treatment. According to the assumptions described in above scenario, $\mathrm{n}=914$ patients should be randomised between the two groups. With an assumed drop-out rate of $20 \%$, $\mathrm{n}=1144$ children will need to be randomised.

\section{Benefits and risks}

Benefits

The potential benefit for a participating child is the prevention (or delay in onset) of beta-cell autoantibodies and diabetes, coeliac disease, childhood infections and allergy. For all participating children, including children who receive placebo, testing blood samples will allow early recognition of presymptomatic T1D and coeliac disease before the child shows the typical symptoms, and an appropriate therapy could be started immediately, potentially reducing complications later in life. Furthermore, information about other ongoing prevention trials or available treatments and intervention studies can be given to families.

\section{Risks}

So far, there have been no reports of risks and side effects associated with the use of $B$. infantis. Several studies show that various strains of $B$. infantis have been administered to numerous newborns and especially to premature babies without complications. In breastfed babies, $B$. infantis is one of the most common bacteria found in the intestine. Typical risks or complaints associated will taking a blood sample cannot be excluded. The volume of blood drawn for the trial endpoints is $<1 \%$ of the total blood volume, within the suggested limits from the European guidelines for a paediatric population. ${ }^{38}$

A data safety monitoring board (DSMB) is established. A DSMB is an independent group of experts responsible to safeguard the well-being and safety of the study participants. The DSMB will meet 6 monthly during the intervention phase and 12-monthly during the follow-up phase. Before each DSMB meeting, the DSMB will receive a report with all relevant information on recruitment rate, data completeness and safety data, including beta-cell and transglutaminase autoantibody and diabetes outcomes.

\section{Retention strategies}

A special family friendly retention concept has been developed to make families feel as part of the research team. Special care and support is offered for families who participate in the study and small gifts for the children are given out during the visits. Families are reminded of the 
advantages of study participation such as free monitoring of the children's health status and potential benefit from the intervention. Strategies for retention also include newsletters and reports on islet and coeliac autoantibody testing, and activities on community building (Homepage, Facebook groups).

Further information can be found on the GPPAD homepage: https://www.gppad.org/de-en/prevention-sintla/

\section{ETHICS AND DISSEMINATION \\ Ethics}

The study was approved by the local ethical committees and regulatory authorities of the Technische Universität München, Medical Faculty (715/20 S), the Technische Universität Dresden (SR+BO-44012021), the Medizinische Hochschule Hannover (9589_BO_S_2021), the Medical University of Warsaw (KB/5/2021), EC Research UZ Leuven (322) and the Swedish ethical review authority (dnr 2021-01210).

\section{Dissemination}

GPPAD is committed to sharing of data in compliance with all applicable European and GPPAD Consortium Member State, Data Protection and Privacy Protection laws, rules and regulations.

Pseudonymised data of the SINT1A Study (GPPAD04 ) will be available to the scientific community after the publication of the trial analysis, which is anticipated in 2028. The SINT1A data will be available on request.

\section{Update on the current status}

The first participant was enrolled in April 2021. By October 2021, six study sites in Germany, Poland, Belgium and Sweden had been initiated stepwise and 155 participants have been enrolled.

\section{Author affiliations}

${ }^{1}$ Institute of Diabetes Research, Helmholtz Zentrum München, Neuherberg, Germany

${ }^{2}$ Forschergruppe Diabetes, Klinikum rechts der Isar, Technische Universität München, Medical Faculty, Munich, Germany

${ }^{3}$ Department of Pediatrics, University Hospital Carl Gustav Carus, Technische Universität Dresden, Dresden, Germany

${ }^{4}$ Center for Regenerative Therapies Dresden (CRTD), Faculty of Medicine, Technische Universität Dresden, Dresden, Germany

${ }^{5}$ Department of Pedriatrics, University Hospitals Leuven, Leuven, Belgium ${ }^{6}$ Department of Development and Regeneration, KU Leuven, Leuven, Belgium ${ }^{7}$ Department of Paediatrics, Skåne University Hospital, Malmö, Sweden

${ }^{8}$ Department of Paediatrics, Skåne University Hospital Lund, Lund, Sweden ${ }^{9}$ Institut für Medizinische Informationsverarbeitung, Biometrie und Epidemiologie, Ludwig-Maximilians-Universität München, Munich, Germany

${ }^{10}$ Kinder- und Jugendkrankenhaus AUF DER BULT, Hannover, Germany

${ }^{11}$ Institute of Mother and Child, Warszawa, Poland

${ }^{12}$ Wellcome Centre for Human Genetics, Nuffield Department of Medicine, NIHR Biomedical Research Centre, University of Oxford, Oxford, UK

${ }^{13}$ Department of Paediatrics, University of Oxford, Oxford, UK

${ }^{14}$ NIHR Oxford Biomedical Research Centre, Oxford University Hospitals NHS Trust, Oxford, UK

${ }^{15}$ Department of Paediatrics, Medical University of Warsaw, Warsaw, Poland
Acknowledgements We highly acknowledge the following support: Data Safety and Monitoring Board: Polly Bingley (University of Bristol, Bristol, UK), Ulrich Heininger (Division of Paediatric Infectious Diseases and Vaccinology, University Children's Hospital, Basel, Switzerland), Markus Pfirrmann (Institut für Medizinische Informationsverarbeitung, Biometrie und Epidemiologie (IBE), Ludwig-MaximiliansUniversität, Munich, Germany), Wolfgang Rascher (Department of Pediatrics and Adolescent Medicine, Erlangen, Germany), Paul Turner (Nuffield Department of Medicine, Medical Science Division, Oxford, UK). Beta-cell autoantibody laboratories: Institute of Diabetes Research, Helmholtz Zentrum München, Munich, Germany. Bristol Medical School, The University of Bristol, Bristol, UK. Genotyping Laboratory: Grace London, LGC, Hertfordshire, UK. Study management/ monitoring: Helmholtz Zentrum München, Neuherberg, Germany. Database and electronic questionnaires: PHARMALOG GmbH, Ismaning, Germany. Study product: Evolve BioSystems, Davis, California, USA. Study pharmacy: Pharmacy of the University Hospital of Heidelberg, Germany. Biorepository: Integrated BioBank of Luxembourg (IBBL), Dudelange, Luxembourg. We thank participating families for their participation in type 1 diabetes research and for helping to develop therapies for prevention.

Collaborators GPPAD-Coordinating Center (CC): Melanie Gündert, Florian Haupt, Stefanie Arnolds, Robin Assfalg, Corinna Barz, Karina Blasius, Nadine Friedl, Cigdem Gezginci, Adriano Gomez-Bantel, Joerg Hasford, Martin Heigermoser, Bianca Höfelschweiger, Manja Jolink, Nadine Klein, Ramona Lickert, Claudia Matzke, Rebecca Niewöhner, Marlon Scholz, Katharina Schütte-Borkovec, Mira Taulien, Lara Vogel, Franziska Voß, Andreas Weiß, José Maria Zapardiel Gonzalo, Philipp Sifft, Heidi Kapfelsberger, Merve Vurucu, Katharina Sarcletti, Stefanie Jacobson, Yulia Grinin. Protocol Committee: John A. Todd, Anette-G. Ziegler (Chairs), Peter Achenbach, Stefanie Arnolds, Reinhard Berner, Ezio Bonifacio, Kristina Casteels, Markus Lundgren, Joerg Hasford, Olga Kordonouri, Marcin L. Pekalski, Matthew Snape, Agnieszka Szypowska. Medical Monitor: Katharina Warncke. Eligibility Committee: Ezio Bonifacio, Joerg Hasford, John A Todd. Outcome Committee: Peter Achenbach, Ezio Bonifacio. Type 1 diabetes endpoint committee: Helena Elding Larsson, Peter Achenbach, Olga Kordonouri, Ezio Bonifacio, Anette G. Ziegler. Belgium Clinical Center: Kristina Casteels, Annre Rochtus, An Jacobs, Hilde Morobé, Jasmin Paulus, Brontë Vrancken, Natalie Van den Driessche, Renka Van Heyste, Janne Houben, Veerle Vanhuyse. Germany, Dresden Clinical Center: Ezio Bonifacio, Reinhard Berner, Sevina Dietz, Gita Gemulla, Manja GottschalkSchwarz, Sophie Heinke, Angela Hommel, Susann Kowal, Fabian Lander, Robert Morgenstern, Marc Weigelt, Nicole Zubizarreta, Sari Arabi, Raphael Hoffmann, Ruth Blechschmidt, Franziska Ehrlich, Anja Loff. Germany, Hanover Clinical Center: Olga Kordonouri, Thomas Danne, Laura Galuschka, Ute Holtkamp, Nils Janzen, Sarah Landsberg, Karin Lange, Erika Marquardt, Felix Reschke, Frank Roloff, Kerstin Semler, Thekla von dem Berge. Germany, Munich Clinical Center: Anette G. Ziegler, Peter Achenbach, Melanie Bunk, Simone Färber-Meisterjahn, Willi Grätz, Ines Greif, Melanie Herbst, Anna Hofelich, Annika Kölln, Benjamin Marcus, Annette Munzinger, Jasmin Ohli, Claudia Ramminger, Franziska Reinmüller, Tiziana Welzhofer, Christiane Winkler. Poland Clinical Center: Agnieszka Szypowska, Mariusz Ołtarzewski, Sylwia Dybkowska, Katarzyna Dżygało, Lidia Groele, Dorota Owczarek, Katarzyna Popko, Agnieszka Skrobot, Anna Taczanowska, Beata Zduńczyk. Sweden Clinical Center: Helena Elding Larsson, Markus Lundgren, Åke Lernmark, Daniel Agardh, Carin Andrén Aronsson, Rasmus Bennet, Charlotte Brundin, Lina Fransson, Ida Jönsson, Sara Maroufkhani, Zeliha Mestan, Caroline Nilsson, Anita Ramelius, Evelyn Tekum Amboh, Carina Törn. UK, Cambridge Clinical Center: Loredana Marcovecchio, Katarzyna Gajewska-Knapik, Sarah Hogg, Elena Romero, Suzannah Twiss. UK, Newcastle Clinical Center: Catherine Owen, Helen Gallon, Laura Gebbie, Fiona Jenkinson. Steven Pratt, Steve Robson, Claire Simmister, Evelyn Thomson, Eileen Walton, Andrew Johnston.

Contributors A-GZ, MLP and JAT conceived the study, and led the protocol team. All authors (A-GZ, SA, AK, PA, RB, EB, KC, HEL, MG, JH, OK, ML, MO, MLP, MP, MDS, AS and JAT) contributed to protocol development. EB, JH and MP developed the statistical design for the study and wrote the statistical section of the protocol. EB and PA developed the outcome definition of the trial and wrote the autoantibody and outcome section of the protocol. A-GZ, SA, AK and $M G$ drafted the manuscript. All authors reviewed the protocol as well as this manuscript.

Funding The SINT1A study is supported by The Leona M. and Harry B. Helmsley Charitable Trust (Helmsley) Grants \#2007-04031 ('The GPPAD-04 study: Supplementation with B. Infantis for Mitigation of Type 1 Diabetes Autoimmunity (SINT1A)'), and by the Helmholtz Zentrum München, Germany. The BMBF trial grant FKZ: 01KX1818 supported advanced genetic risk assessment and trial protocol development phase. The German Clinical Centers Munich and Dresden are also supported by the Deutsches Zentrum für Diabetesforschung DZD. 
Disclaimer Funding organisations had no role in the design of the trial.

Competing interests JAT is a member of the Scientific Advisory Board of Precion and of the Human Genetics Advisory Board of GSK

Patient consent for publication Not applicable.

Provenance and peer review Not commissioned; externally peer reviewed.

Supplemental material This content has been supplied by the author(s). It has not been vetted by BMJ Publishing Group Limited (BMJ) and may not have been peer-reviewed. Any opinions or recommendations discussed are solely those of the author(s) and are not endorsed by BMJ. BMJ disclaims all liability and responsibility arising from any reliance placed on the content. Where the content includes any translated material, BMJ does not warrant the accuracy and reliability of the translations (including but not limited to local regulations, clinical guidelines, terminology, drug names and drug dosages), and is not responsible for any error and/or omissions arising from translation and adaptation or otherwise.

Open access This is an open access article distributed in accordance with the Creative Commons Attribution Non Commercial (CC BY-NC 4.0) license, which permits others to distribute, remix, adapt, build upon this work non-commercially, and license their derivative works on different terms, provided the original work is properly cited, appropriate credit is given, any changes made indicated, and the use is non-commercial. See: http://creativecommons.org/licenses/by-nc/4.0/.

\section{ORCID iD}

Melanie Gündert http://orcid.org/0000-0003-2236-6604

\section{REFERENCES}

1 Ziegler AG, Rewers M, Simell O, et al. Seroconversion to multiple islet autoantibodies and risk of progression to diabetes in children. JAMA 2013;309:2473-9.

2 Ziegler A-G, Bonifacio E, BABYDIAB-BABYDIET Study Group. Agerelated islet autoantibody incidence in offspring of patients with type 1 diabetes. Diabetologia 2012;55:1937-43.

3 Kimpimäki T, Kulmala P, Savola K, et al. Natural history of beta-cell autoimmunity in young children with increased genetic susceptibility to type 1 diabetes recruited from the general population. J Clin Endocrinol Metab 2002;87:4572-9.

4 Krischer JP, Lynch KF, Schatz DA, et al. The 6 year incidence of diabetes-associated autoantibodies in genetically at-risk children: the TEDDY study. Diabetologia 2015;58:980-7.

5 Hoffmann VS, Weiß A, Winkler C, et al. Landmark models to define the age-adjusted risk of developing stage 1 type 1 diabetes across childhood and adolescence. BMC Med 2019;17:125.

6 Bonifacio E, Weiß A, Winkler C, et al. An age-related exponential decline in the risk of multiple islet autoantibody seroconversion during childhood. Diabetes Care 2021:dc202122.

7 Winkler C, Haupt F, Heigermoser M, et al. Identification of infants with increased type 1 diabetes genetic risk for enrollment into primary prevention Trials-GPPAD-02 study design and first results. Pediatr Diabetes 2019;20:720-7.

8 Ziegler A-G, Achenbach P, Berner R, et al. Oral insulin therapy for primary prevention of type 1 diabetes in infants with high genetic risk: the GPPAD-POInT (global platform for the prevention of autoimmune diabetes primary oral insulin trial) study protocol. BMJ Open 2019;9:e028578.

9 Endesfelder D, zu Castell W, Ardissone A, et al. Compromised gut microbiota networks in children with anti-islet cell autoimmunity. Diabetes 2014;63:2006-14.

10 Vatanen T, Franzosa EA, Schwager R, et al. The human gut microbiome in early-onset type 1 diabetes from the TEDDY study. Nature 2018;562:589-94.

11 Uusitalo U, Liu X, Yang J, et al. Association of early exposure of probiotics and islet autoimmunity in the TEDDY study. JAMA Pediatr 2016;170:20-8.

12 Garcia AR, Paterou A, Lee M. Peripheral tolerance to insulin is encoded by mimicry in the microbiome. bioRxiv 2019.

13 Hsiao K-C, Ponsonby A-L, Axelrad C, et al. Long-term clinical and immunological effects of probiotic and peanut oral immunotherapy after treatment cessation: 4-year follow-up of a randomised, double-blind, placebo-controlled trial. Lancet Child Adolesc Health 2017;1:97-105.

14 Panigrahi P, Parida S, Nanda NC, et al. A randomized synbiotic trial to prevent sepsis among infants in rural India. Nature 2017;548:407-12.
15 Mariño E, Richards JL, McLeod KH, et al. Gut microbial metabolites limit the frequency of autoimmune T cells and protect against type 1 diabetes. Nat Immunol 2017;18:552-62.

16 Sommer F, Bäckhed F. The gut microbiota--masters of host development and physiology. Nat Rev Microbiol 2013;11:227-38.

17 Stewart CJ, Ajami NJ, O'Brien JL, et al. Temporal development of the gut microbiome in early childhood from the TEDDY study. Nature 2018;562:583-8.

18 Amenyogbe N, Kollmann TR, Ben-Othman R. Early-life hostmicrobiome interphase: the key frontier for immune development. Front Pediatr 2017;5:111.

19 Vatanen T, Kostic AD, d'Hennezel E, et al. Variation in microbiome LPS immunogenicity contributes to autoimmunity in humans. Cell 2016;165:842-53.

20 Davis-Richardson AG, Ardissone AN, Dias R, et al. Bacteroides dorei dominates gut microbiome prior to autoimmunity in Finnish children at high risk for type 1 diabetes. Front Microbiol 2014;5:678.

21 Kostic AD, Gevers D, Siljander $\mathrm{H}$, et al. The dynamics of the human infant gut microbiome in development and in progression toward type 1 diabetes. Cell Host Microbe 2015;17:260-73.

22 Russell JT, Roesch LFW, Ördberg M, et al. Genetic risk for autoimmunity is associated with distinct changes in the human gut microbiome. Nat Commun 2019;10:3621.

23 Chichlowski M, Shah N, Wampler JL, et al. Bifidobacterium longum Subspecies infantis ( $B$. infantis) in pediatric nutrition: current state of knowledge. Nutrients 2020;12:1581.

24 Insel R, Knip M. Prospects for primary prevention of type 1 diabetes by restoring a disappearing microbe. Pediatr Diabetes 2018;19:1400-6.

25 Ismail IH, Boyle RJ, Licciardi PV, et al. Early gut colonization by Bifidobacterium breve and B. catenulatum differentially modulates eczema risk in children at high risk of developing allergic disease. Pediatr Allergy Immunol 2016;27:838-46.

26 van der Aa LB, van Aalderen WMC, Heymans HSA, et al. Synbiotics prevent asthma-like symptoms in infants with atopic dermatitis. Allergy 2011;66:170-7.

27 Huda MN, Ahmad SM, Alam MJ, et al. Bifidobacterium abundance in early infancy and vaccine response at 2 years of age. Pediatrics 2019;143:e20181489.

28 Smilowitz J, Moya J, Breck M. Safety and tolerability of Bifidobacterium longum subspecies infantis EVC001 supplementation in healthy term breastfed infants: a phase I clinical trial. BMC Pediatrics 2017;17.

29 Frese SA, Hutton AA, Contreras LN, et al. Persistence of Supplemented Bifidobacterium longum subsp. infantis EVC001 in Breastfed Infants. mSphere 2017;2:e00501-17.

30 Henrick BM, Chew S, Casaburi G, et al. Colonization by B. infantis EVC001 modulates enteric inflammation in exclusively breastfed infants. Pediatr Res 2019;86:749-57.

31 Ziegler AG, Danne T, Dunger DB, et al. Primary prevention of betacell autoimmunity and type 1 diabetes - The Global Platform for the Prevention of Autoimmune Diabetes (GPPAD) perspectives. Mol Metab 2016;5:255-62.

32 Hippich M, Beyerlein A, Hagopian WA, et al. Genetic contribution to the divergence in type 1 diabetes risk between children from the general population and children from affected families. Diabetes 2019;68:847-57.

33 Liu E, Lee H-S, Aronsson CA, et al. Risk of pediatric celiac disease according to HLA haplotype and country. N Engl J Med 2014:371:42-9.

34 Bonifacio E, Beyerlein A, Hippich M, et al. Genetic scores to stratify risk of developing multiple islet autoantibodies and type 1 diabetes: a prospective study in children. PLoS Med 2018;15:e1002548.

35 Duar RM, Casaburi G, Mitchell RD, et al. Comparative Genome Analysis of Bifidobacterium longum subsp. infantis Strains Reveals Variation in Human Milk Oligosaccharide Utilization Genes among Commercial Probiotics. Nutrients 2020;12:3247.

36 Smyth DJ, Cooper JD, Howson JMM, et al. Fut2 nonsecretor status links type 1 diabetes susceptibility and resistance to infection. Diabetes 2011;60:3081-4.

37 Yang P, Li H-L, Wang C-Y. FUT2 nonfunctional variant: a "missing link" between genes and environment in type 1 diabetes? Diabetes 2011;60:2685-7

38 Online referencing, 2017. Available: http://ec.europa.eu/health// sites/health/files/files/eudralex/vol-10/ethical_considerations_en.pdf [Accessed 12 Jun 2017]. 